Check for updates

Cite this: RSC Adv., 2017, 7, 18260

Received 24th January 2017

Accepted 14th March 2017

DOI: $10.1039 / c 7 r a 01082 f$

rsc.li/rsc-advances

\section{Conductive biofoams of wheat gluten containing carbon nanotubes, carbon black or reduced graphene oxide $\uparrow$}

\author{
Qiong Wu, Henrik Sundborg, Richard L. Andersson, (D) Kevin Peuvot, Léonard Guex, \\ Fritjof Nilsson, Mikael S. Hedenqvist and Richard T. Olsson (D)*
}

\begin{abstract}
Conductive biofoams made from glycerol-plasticized wheat gluten (WGG) are presented as a potential substitute in electrical applications for conductive polymer foams from crude oil. The soft plasticised foams were prepared by conventional freeze-drying of wheat gluten suspensions with carbon nanotubes (CNTs), carbon black (CB) or reduced graphene oxide (rGO) as the conductive filler phase. The change in conductivity upon compression was documented and the results show not only that the CNT-filled foams show a conductivity two orders of magnitude higher than foams filled with the CB particles, but also that there is a significantly lower percolation threshold with percolation occurring already at 0.18 vol\%. The rGO-filled foams gave a conductivity inferior to that obtained with the CNTs or CB particles, which is explained as being related to the sheet-like morphology of the rGO flakes. An increasing amount of conductive filler resulted in smaller pore sizes for both CNTs and CB particles due to their interference with the ice crystal formation before the lyophilization process. The conductive WGG foams with CNTs were fully elastic with up to $10 \%$ compressive strain, but with increasing compression up to $50 \%$ strain the recovery gradually decreased. The data show that the conductivity strongly depends on the type as well as the concentration of the conductive filler, and the conductivity data with different compressions applied to these biofoams are presented for the first time.
\end{abstract}

\section{Introduction}

A flexible conducting polymer composite (CPC) made from a lightweight porous foam and a conductive filler exhibits interesting electrical properties, e.g. the conductivity changes when the material is mechanically strained/compressed. Possible applications include switches or sensors that are triggered by the mechanical deformation, as well as packaging materials for electrostatic discharge protection (ESD) and electromagnetic interference (EMI) shields. ${ }^{1-3}$ According to the standards, JEDEC625B ${ }^{4}$ and EIA-541, ${ }^{5}$ three levels of conductive materials are defined related to their use within the electronic industry: insulative (less than $10^{-13} \mathrm{~S} \mathrm{~m}^{-1}$ ), static dissipative (between $10^{-6} \mathrm{~S} \mathrm{~m}^{-1}$ and $10^{-13} \mathrm{~S} \mathrm{~m}^{-1}$ ) and conductive (more than $10^{-6} \mathrm{~S} \mathrm{~m}^{-1}$ ). To reach these levels of conductivity in a CPC foam, electron percolation between conductive filler particles present in the cell walls of the porous composites has to occur. Conductive carbon-based filler particles can here be used and as spherical carbon black (CB), large aspect ratio carbon filaments such as carbon fibres, carbon nanotubes (CNTs), or in the future

Department of Fiber and Polymer Technology, Royal Institute of Technology, SE-10044 Stockholm, Sweden. E-mail: rols@kth.se

$\dagger$ Electronic supplementary information (ESI) available. See DOI: $10.1039 / \mathrm{c} 7 \mathrm{ra0} 082 \mathrm{f}$ possibly the recently commercialized graphene, which is attracting a growing interest. ${ }^{6-9}$ Among these, the CNTs show many advantages not only due to their high conductivity and high aspect ratio (making percolation at very low concentration possible), but also to their ability to simultaneously reinforce the composite. ${ }^{\mathbf{1 0}}$ The use of high aspect ratio carbon-based fillers to produce conductive networks has been reported for some porous CPC systems., ${ }^{\mathbf{1 1 1 - 1 5}}$ From these studies, it can be concluded that a predictable dispersion of the carbon filler is always required to achieve an electron percolation at a very low concentration $(0.2 \mathrm{vol} \%)^{16}$ in the otherwise insulating polymer matrix. ${ }^{17}$ CPCs with small amounts of carbon filler (0.005-0.1 vol\% $\%)^{18}$ also exist as phase-segregated polymer systems, where one phase carries the conductive carbon based filler, but these systems have not yet been reported for porous CPCs. ${ }^{19-23}$

The attention to porous CPCs has so far mainly been devoted to traditional polymeric materials derived from petroleum resources. However, polymeric materials from renewable resources are growing in numbers and several bio-CPCs are expected in the future. The difference between the traditional polymers from petroleum and those from a renewable resource is generally the higher hydrophilicity of the latter, which affects their solidification and interactive chemistry in nature, often involving condensation reactions. Examples of biofoams include those based on polysaccharides (e.g. cellulose, ${ }^{24}$ 
chitosan ${ }^{25}$ and chitin ${ }^{26}$ ), which are materials typically extracted from forest or marine resources ${ }^{27,28}$ and those based on proteins (e.g. soy, collagen, silk, and wheat). Wheat gluten from wheat is a particularly interesting protein for foam applications, since it has good cohesion and can easily be foamed under ambient conditions, due to its ability to polymerize in solution. ${ }^{29}$ The high cohesion arises from a high molar mass (high content of chain entanglements), the ability to form cross-links (disulfide and isopeptide bonds) and a high content of hydrogen bonds. Wheat gluten is available in large volumes as an agricultural co/ by-product from e.g. the bio-ethanol industry, ${ }^{30}$ and can be tailored to various mechanical properties using a glycerol plasticiser. ${ }^{31}$ Since glycerol is a large by-product from biodiesel production, ${ }^{32}$ the combination of WG and glycerol is a cheap biobased material derived from industrial side-streams.

In our previous studies, we have developed foams with a range of properties where the solid material formation and porosity were achieved by freeze-drying an aqueous solution of wheat gluten..$^{31,33-35}$ In this paper, we report for the first time that WG foams can be converted into highly porous and conductive biofoams by the addition of carbon nanotubes (CNTs), and these foams are compared with those containing carbon black nanoparticles (CB). The most conductive foams were those containing CNTs. For comparison, a sample containing reduced graphene oxide ( $\mathrm{rGO}$ ) was made with a concentration of $c a .0 .4$ $\mathrm{vol} \%$, which corresponded to the weight/volume fraction where both the CNT and CB filler systems showed percolation. The electrical properties of the conductive biofoams were then measured under compression within a developed instrumental setup for simultaneous measurements. To our best knowledge, this has never been reported before for conductive biofoams. The measurements revealed that the conductivity of the CB and CNT filled foams decreased with increasing compressive strain, whereas the conductivity of the rGO-filled foam first decreased and then increased. It is suggested that this difference in conductivity behaviour during compression is primarily due to the different particle morphologies and their different aspect ratios. Since the resistances of the CNT and CB filled foams were essentially independent of the degree of compression, these materials appear suitable for use in electromagnetic shielding (EMI) and electrostatic discharge (ESD) applications.

\section{Experimental}

\subsection{Materials}

Commercial wheat powder (WG) was supplied by Lantmännen Reppe AB, Sweden. The WG protein content was $78 \mathrm{wt} \%$ and the starch content was $5.8 \mathrm{wt} \%$, as determined by the Ewers Polarimetric method. Ultrapure HPLC grade glycerol (99.5\%) was purchased from Alfa Aesar. Sodium hydroxide (puriss. p.a ACS $>98 \%)$, reagent grade sodium borohydride $\left(\mathrm{NaBH}_{4}\right)$ and technical grade sodium dodecyl benzene sulfonate (SDBS) were purchased from Sigma Aldrich. Ammonium hydroxide solution of $30 \%$ (puriss. p.a) was purchased from Fluka and was used as an alkaline buffer solution to prevent aqueous depletion (hydrolysis) of the $\mathrm{NaBH}_{4}$ during the reduction of the graphene oxide $^{36}{ }^{36}$ Flo Tube ${ }^{\mathrm{TM}} 7000$ Vertically Aligned Carbon Nano Tubes
(CNT, purity $>93 \%$ ) were purchased from Cnano Technology Limited. The CNTs are multi-walled (6-8 nm wall thickness), with dimensions up to $40 \mu \mathrm{m}$ long and $20 \mathrm{~nm}$ thick $\left(A_{\mathrm{r}}=2000\right)$, as shown in Fig. 1a. The carbon black (CB) was supplied by Orion Engineered Carbons as Printex XE 2B and consisted of carbon particles $20 \mathrm{~nm}$ in diameter. Fig. $1 \mathrm{~b}$ shows a TEM image of the CB aggregates. Graphene oxide (GO) was purchased as an aqueous suspension ( $0.5 \mathrm{wt} \%$ solid GO content, N002-PS-0.5) from Angstrom Materials, Dayton (USA). The lateral $x / y$ dimensions were $c a .5000 \mathrm{~nm}$ (measured from TEM) and thickness was 1-1.2 $\mathrm{nm}$ (according to the manufacturer's AFM measurements).
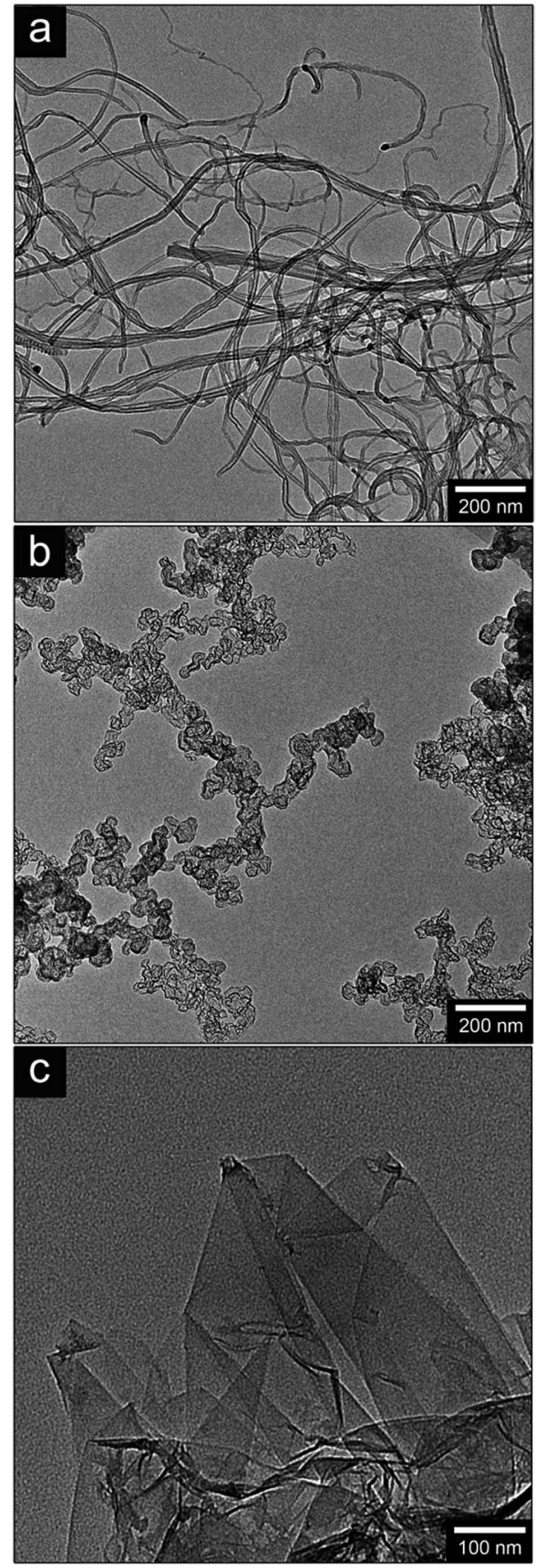

Fig. 1 TEM images of (a) CNT, (b) CB and (c) rGO. 


\subsection{Preparation of reduced graphene oxide (rGO)}

A $120 \mathrm{ml}$ alkaline buffer solution was prepared using $76.7 \mathrm{ml}$ of Milli-Q water, $43.3 \mathrm{ml}$ of a $10 \mathrm{vol} \% \mathrm{NaOH}$ buffer solution and $2.27 \mathrm{~g}$ of $\mathrm{NaBH}_{4}$ reducing agent. The chemical reduction was carried out using $80 \mathrm{ml}$ of graphene oxide suspension and $120 \mathrm{ml}$ of the alkaline solution under magnetic stirring in sealed borosilicate glassware, placed in a silicone oil bath at $85{ }^{\circ} \mathrm{C}$ for 24 hours. The resulting suspension was centrifuged at $4000 \mathrm{rpm}$ for $30 \mathrm{~min}$ and the supernatant liquid was changed to Milli/Q water. This exchange was repeated three times to eliminate residual ions from the salts. The morphology of rGO is shown in Fig. 1c. After reduction, the conductivity of the rGO sheets was found to be $1850 \mathrm{~S} \mathrm{~m}^{-1}$ by 4-probe measurements on an ensemble of sheets on a nonconductive surface.

\subsection{Preparation of glycerol-plasticised WG foam}

$5 \mathrm{~g}$ of WG powder was slowly added to $50 \mathrm{ml}$ Milli-Q water under magnetic stirring. The $\mathrm{pH}$ of the dispersion was then adjusted to 11 by dropwise addition of $1 \mathrm{M} \mathrm{NaOH}$ aqueous solution, and then stirred for $10 \mathrm{~min}$. The total volume of Milli-Q water was then adjusted to $60 \mathrm{ml}$, and $2.16 \mathrm{~g}$ of glycerol was added. The beaker containing the mixture was placed in a silicone oil bath and heated from room temperature to $90{ }^{\circ} \mathrm{C}$ at a rate of $c a .7{ }^{\circ} \mathrm{C}$ $\min ^{-1}$, under continuous magnetic stirring. The mixture was then cooled to room temperature and poured into cuboid silicone moulds with dimensions of $10 \times 10 \times 11(\mathrm{~mm})^{3}$. The moulds containing the mixtures were then frozen at $-25{ }^{\circ} \mathrm{C}$ for at least $6 \mathrm{~h}$ before being freeze-dried for $24 \mathrm{~h}$ (ScanVac CoolSafe freeze-dryer). The final foam was named WGG.

\subsection{Preparation of WGG foams containing CNT, CB or rGO}

$0.13 \mathrm{~g}$ of the surfactant SDBS was dissolved in $50 \mathrm{ml}$ of Milli-Q water under magnetic stirring, and $0.13 \mathrm{~g}$ of CNTs was slowly added under magnetic stirring. The mass ratio of SDBS to CNT was kept at $1: 1$ in all the samples. The mixture was sonicated for $10 \mathrm{~min}$ in an ultrasonic bath (Branson 2510DTH), and $5 \mathrm{~g}$ of WG powder was then added slowly to the mixture under magnetic stirring. The $\mathrm{pH}$ of the suspension was adjusted to 11 by dropwise addition of $1 \mathrm{M} \mathrm{NaOH}$ aqueous solution. The total volume of water was then adjusted to $60 \mathrm{ml}, 2.2 \mathrm{~g}$ of glycerol was added and the mixture was stirred for $10 \mathrm{~min}$ before being heated to $90{ }^{\circ} \mathrm{C}$. The rest of the preparation was the same as that of the WGG foam. This composition resulted in $0.15 \mathrm{vol} \%$ CNTs in the final freeze-dried foam (equal to 2.5 $\mathrm{wt} \% \mathrm{CNT}$ in the CNT/WG mixture), and this foam is referred to as $\mathrm{CNT}(0.15)$. When $\mathrm{CB}$ or rGO was used as conductive filler, the preparation followed the same procedure as with the CNT(0.15) foam, the only difference being the filler type and the amount used. For comparison, a foam with 0.39 vol\% rGO was prepared ( $\mathrm{rGO}(0.39))$. Table 1 shows the composition of all the samples. All the samples were made from $60 \mathrm{ml}$ Milli-Q water, $5 \mathrm{~g}$ WG and $2.2 \mathrm{~g}$ glycerol and are named according to the filler type and volume percentage of conductive filler used.
Table 1 Foam compositions

\begin{tabular}{llc}
\hline Sample & $\begin{array}{l}\text { Filler } \\
\text { content }^{a}(\text { vol } \%)\end{array}$ & $\begin{array}{l}\text { Filler } \\
\text { content }^{b}\left(\text { wt }^{2}\right)\end{array}$ \\
\hline WGG & 0 & 0 \\
CNT $(0.15)$ & 0.15 & 2.5 \\
CNT $(0.18)$ & 0.18 & 3.0 \\
CNT $(0.20)$ & 0.20 & 3.5 \\
CNT $(0.22)$ & 0.22 & 4.0 \\
CNT $(0.24)$ & 0.24 & 4.5 \\
CNT $(0.26)$ & 0.26 & 4.8 \\
CNT $(0.41)$ & 0.41 & 10.1 \\
CNT $(0.49)$ & 0.49 & 15.0 \\
CB $(0.27)$ & 0.27 & 4.8 \\
CB $(0.44)$ & 0.44 & 10.1 \\
CB $(0.53)$ & 0.53 & 15.0 \\
rGO $(0.39)$ & 0.39 & 10.1 \\
a Filler content in volume percentage of the foam. ${ }^{b}$ Filler content in \\
weight percentage of the filler-WG weight.
\end{tabular}

\subsection{Transmission electron microscope (TEM)}

TEM images of CNTs, CB and rGO were obtained using a Hitachi HT7700 microscope operated at $100 \mathrm{kV}$. Samples of particles were deposited onto holey carbon-coated 400 mesh copper grids (TED PELLA, USA) from ultrasonicated suspensions of particles dispersed in ethanol.

\subsection{Field-emission scanning electron microscope (FE-SEM)}

The morphology of the foams was studied using a Hitachi S4800 field emission scanning electron microscope. Prior to observation, the samples were coated with platinum/palladium (60/40) for 20 seconds using a Cressington high-resolution sputter coater (model 208RH). The pore size was determined by Image 1.50 b (Wayne Rasband National Institutes of Health, USA) from the 2D SEM images as the largest diameter of each pore. A minimum of 50 pores was determined for each sample.

\subsection{Mechanical and electrical measurements}

Compression testing was performed in an Instron 5566 universal testing machine with a $500 \mathrm{~N}$ load cell. Compression plates (T1223-1021) with a diameter of $50 \mathrm{~mm}$ were used. All the specimens were conditioned at $23 \pm 1{ }^{\circ} \mathrm{C}$ and $50 \pm 2 \% \mathrm{RH}$ for at least $120 \mathrm{~h}$ prior to measurement. The conductivity was measured simultaneously during the compression testing by a Keithley 2450 SourceMeter SMU (source measure unit) connected to two parallel conductive copper plates attached directly to the sample with a layer of conductive colloidal graphite paste (TedPella, USA) between the copper plates and the sample, as shown in Fig. 2. A thin insulated copper wire $(0.36 \mathrm{~mm}$ outer diameter) was soldered onto the copper plates and the wires were connected to the SMU. The resistance of the wires and the adhesive was negligible compared to the resistance of the foam, and a $0.075 \mathrm{~mm}$ thick polyethylene film was used as galvanic insulation between the metallic compression plates and the sample. The dimensions of the specimens were measured with a digital caliper prior to and after the compression, and the 
(a) Sample preparation

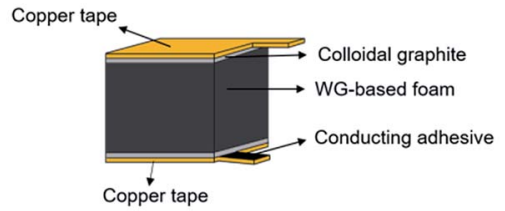

(b) Compression testing and resistance measurement

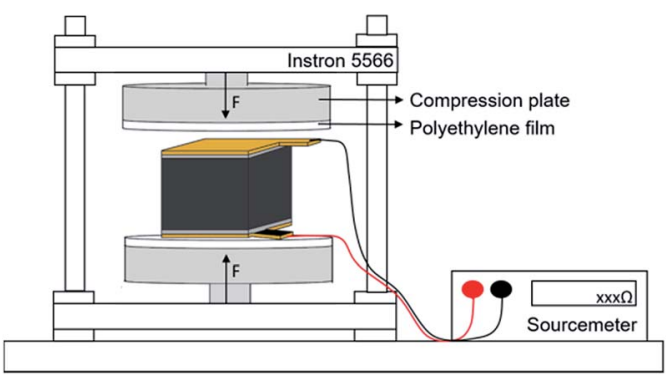

Fig. 2 Compression testing of foam with simultaneous resistance measured.

initial dimension of the specimen were $10 \times 10 \times 11(\mathrm{~mm})^{3}$. The deformation rate was $10 \% \mathrm{~min}^{-1}$ (based on the original specimen thickness), which was equivalent to $1 \mathrm{~mm} \mathrm{~min}^{-1}$. Cyclic compression testing was performed using a total of 5 subsequent cycles on the same specimen. In the first cycle, the foam was compressed to $10 \%$ of its thickness, after which the compression plates were returned to the initial position $(0 \%$ strain), and kept there for $1 \mathrm{~min}$, to allow the foam to recover from the compressed state. This cycle was repeated but with maximum strains of, consecutively $20,30,40$ and $50 \%$. The compressive strain is shown as a function of time in the ESI (Fig. S1†).

\section{Results and discussion}

\subsection{Morphology of WGG foams with CNT, CB and rGO}

Fig. 3 shows cross sections of the glycerol-plasticised wheat gluten foams (WGG) with different amount of CNTs, CB or rGO together with the virgin WGG foam. The virgin WGG foam had a lamellar cell structure, which originated from the directional growth of ice crystals during the freezing of the WGG aqueous mixture prior to the freeze-drying (Fig. 3a). The lamellar structure became less elongated with the presence of the fillers at contents $>0.3$ vol\% ( $c f$. Fig. $3 \mathrm{a}$ and $\mathrm{c}-\mathrm{f}$ ). The elongation/ anisotropy decreased with increasing CNT and CB contents (not shown for CB). It is suggested that the decrease in cell anisotropy is due to the filler particles acting as nucleation points for ice-formation. At the highest concentrations of CNTs ( 0.41 and 0.49 vol\%), (Fig. $3 \mathrm{c}$ and $\mathrm{d}$ ), the lamellar structure had transformed into a morphology that was more like bordering ellipsoidal spheres. It is suggested, in addition to the nucleation effect, that this was also due to a higher solution viscosity with increasing amounts of fillers, which made it more difficult for the water to migrate to the growing ice crystal. Fig. $3 \mathrm{c}$ and $\mathrm{d}$ also show that agglomerates of WGG/CNT existed at the two highest concentrations of CNTs. The role of these agglomerates in the growth of extended lamellar ice crystals is however unclear. In Table 2, it can be seen that although the average cell size decreased from 1187 to $404 \mu \mathrm{m}$ with increasing CNT content, the density of the materials remained essentially the same (170$190 \mathrm{~kg} \mathrm{~m}^{-3}$ ) whereas the average cell wall thickness was ca. 5-25 $\mu \mathrm{m}$. The porosity was also relatively unaffected by the addition of the CNTs and varied only between 85 and $88 \%$.

Fig. 3e shows the cross sectional morphology of the WGG foam filled with $0.44 \mathrm{vol} \% \mathrm{CB}$ particles. This foam has smaller and less anisotropic cells than the CNT foams, indicating a more
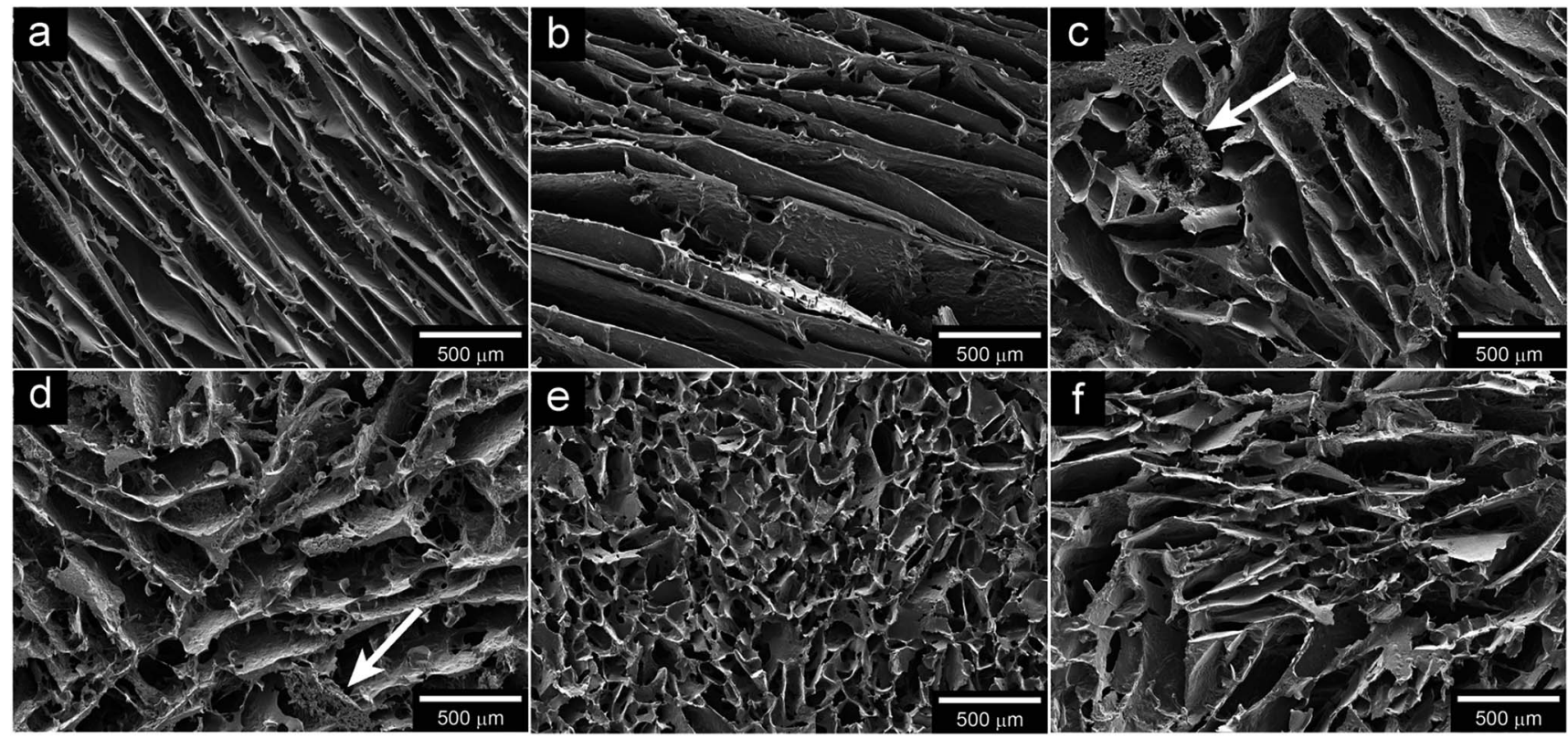

Fig. 3 SEM micrographs of plasticised-WG foam with (a) $0 \%$ filler, (b) 0.26 vol\% CNT, (c) 0.41 vol\% CNT, (d) 0.49 vol\% CNT, (e) 0.44 vol\% CB, (f) 0.39 vol\% rGO. White arrows indicate agglomerates. 
Table 2 Foam properties

\begin{tabular}{llccc}
\hline Sample ID & Density $^{a}\left(\mathrm{~kg} \mathrm{~m}^{-3}\right)$ & ${\text { Pore } \operatorname{size}^{b}(\mu \mathrm{m})}^{c}(\%)$ & $\phi^{d}(\mathrm{kPa})$ & Cond. ratio $^{e}$ \\
\hline WGG & $179 \pm 2$ & $1187 \pm 336$ & 86.1 & $36 \pm 4$ \\
CNT(0.15) & $172 \pm 5$ & $1178 \pm 390$ & 86.2 & $13 \pm 3$ \\
CNT(0.26) & $190 \pm 6$ & $1149 \pm 462$ & 85.3 & $16 \pm 2$ \\
CNT(0.41) & $170 \pm 2$ & $555 \pm 147$ & 87.9 & $33 \pm 8$ \\
CNT(0.49) & $172 \pm 5$ & $404 \pm 156$ & 85.8 & $35 \pm 11$ \\
CB(0.27) & $184 \pm 18$ & $384 \pm 150$ & 86.6 & $18 \pm 12$ \\
CB(0.44) & $189 \pm 17$ & $133 \pm 21$ & 87.5 & 1.6 \\
CB(0.53) & $192 \pm 6$ & $135 \pm 30$ & 88.2 & $21 \pm 7$ \\
rGO(0.39) & $167 \pm 2$ & $561 \pm 146$ & $26 \pm 7$
\end{tabular}

${ }^{a}$ Average density \pm standard deviation from 5 specimens. ${ }^{b}$ Average cell size \pm standard deviation from a minimum 50 measurements, the cell size is the longest diameter of the cell. ${ }^{c}$ Porosity calculated as $\phi=1-\left(\rho_{\text {bulk }} / \rho_{\text {solid }}\right) .{ }^{d}$ Average compression modulus \pm standard deviation from 5 specimens. ${ }^{e}$ Conductivity ratio between 0 and $50 \%$ compressive strain.

significant ice nucleation effect of the carbon black nanoparticles (cf. Fig. 3c and e, and Table 2). It is suggested that this is due to the smaller size of the individual $20 \mathrm{~nm}$ CB particles, with more surface on which ice can nucleate than in the CNT case. In agreement with this suggestion, the nucleation effect of rGO was similar to that of CNTs, as observed from the foams having similar anisotropy and cell size for a given amount of carbon filler of around 0.4 vol\% (cf. Fig. $3 c$ and $\mathrm{f}$, and Table 2). Table 2 also shows that, as the pore size decreased for the $\mathrm{CB}$ foams with increasing CB content, the density increased from $c a$. 180 to 190 $\mathrm{kg} \mathrm{m}^{-3}$. The difference in density was small but stood in contrast to the density values for the rGO filled foams, which showed the lowest densities of all the prepared foams $\left(167 \pm 2 \mathrm{~kg} \mathrm{~m}^{-3}\right)$.

\subsection{Electrical conductivity}

Fig. 4a shows the conductivities of the foams containing different concentrations of CNTs and $\mathrm{CB}$ at $50 \% \mathrm{RH}$. The conductivity of the virgin WGG foam was $c a .1 .6 \times 10^{-4} \mathrm{~S} \mathrm{~m}^{-1}$. This small conductivity was related to the presence of ions and water in the foam. At $c a .0 \% \mathrm{RH}$ the conductivity was $c a .2$ orders of magnitude lower. The conductivity increased $c a$. 3 orders of magnitude with increasing filler concentration for both CNTs and $\mathrm{CB}$ particles, after the percolation threshold had been reached (Fig. 4a). The percolation threshold was significantly higher for the CB system, which required more than 0.40 vol\% particles to display any electrical conductivity, whereas only 0.16-0.18 vol\% CNTs was needed for the CNT system. The percolation thresholds of CNT-filled polymers has been reported to be as low as $0.0042 \mathrm{vol} \%{ }^{16}$ However, a more general value for percolation is in the range of $0.2-1.6 \mathrm{vol} \%$ for polymer nanocomposites containing CNTs (average from more than 40 studies). ${ }^{17}$ This indicates that the dispersion of CNTs in the WGG was successful and that a conductive network was formed in the lower range of the reported percolation thresholds of CNTs. Overall, the conductivities of the CNT foams were always higher than those of the CB foams with the same mass of carbon filler. With 0.4 vol\% filler the conductivity was $0.24 \mathrm{~S}$ $\mathrm{m}^{-1}$ for the CNT system and $0.033 \mathrm{~S} \mathrm{~m}^{-1}$ for the CB system. The highest conductivity $\left(0.88 \mathrm{~S} \mathrm{~m}^{-1}\right)$ was observed for WGG with 0.49 vol\% CNTs. The solid lines in Fig. 4 a represent best fits to the analytical percolation theory equation: ${ }^{37}$

$$
(1-\phi) \frac{\left(\sigma_{\mathrm{m}}{ }^{1 / s}-\sigma^{1 / s}\right)}{\sigma_{\mathrm{m}}{ }^{1 / s}+\sigma^{1 / s}\left(1-\phi_{\mathrm{p}}\right) / \phi_{\mathrm{p}}}+\phi \frac{\left(\sigma_{\mathrm{m}}^{1 / t}-\sigma^{1 / t}\right)}{\sigma_{\mathrm{f}}^{1 / t}+\sigma^{1 / t}\left(1-\phi_{\mathrm{p}}\right) / \phi_{\mathrm{p}}}=0
$$

where $s=0.87$ and $t=2$ are universal constants for a 3D system, $\sigma_{\mathrm{m}}, \sigma_{\mathrm{f}}$ and $\sigma$ are the conductivities of the matrix (WGG), the filler and the composite, respectively. $\phi$ is the volume fraction of the filler and $\phi_{\mathrm{p}}$ is the calculated percolation threshold. In the best fits, the filler conductivity was set at the same value for both CNTs and CB particles: $80000 \mathrm{~S} \mathrm{~m}^{-1}$. The calculated CNT percolation threshold, $\phi_{\mathrm{p}}=0.18 \mathrm{vol} \%$, was however somewhat
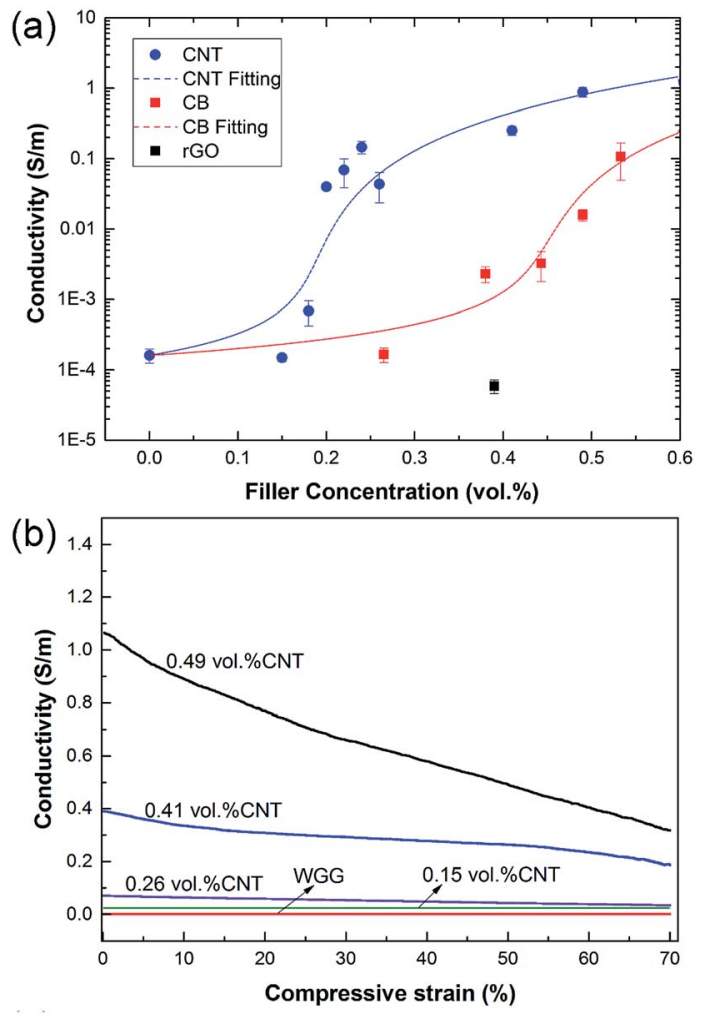

Fig. 4 (a) Conductivity of non-deformed WG foams with different concentrations of CNT and CB and of the rGO foam, (b) conductivity of foams with different concentrations of CNT as a function of compressive strain. 
higher than the theoretical value $\left(\phi_{\mathrm{p}}=0.025 \mathrm{vol} \%\right)$ for an ideally dispersed isotropic fibre composite with an aspect ratio of $A_{\mathrm{r}}=$ 2000 , calculated with the semi-empirical equation: ${ }^{38}$

$$
\phi_{\mathrm{p}}=1 / 2 A_{\mathrm{r}}
$$

The calculated percolation threshold value for the CB/WGG composite of $\phi_{\mathrm{p}}=0.43 \mathrm{vol} \%$ (using eqn (1)) was lower than most of the previous reported values for $\mathrm{CB} /$ polymer nanocomposites, but in relatively good agreement with the measured data. Typical $\phi_{\mathrm{p}}$ values for $\mathrm{CB} /$ polymer nanocomposites are in the range of $1.5-22 \mathrm{vol}^{3.39,40}$ The theoretical percolation threshold for a nanocomposite containing perfectly dispersed spherical CB particles $\left(A_{\mathrm{r}}=1\right)$ in a solid polymer is $c a . \phi_{\mathrm{p}}=29$ vol\% ${ }^{41}$ However, since the percolation of primary CB-particles relies on the formation of conducting strings and aggregates, conduction through the material typically occurs at a $\phi$-value significantly lower than in a perfectly dispersed CB composite. ${ }^{\mathbf{4 2}}$

The sample containing reduced graphene oxide (rGO) showed a significantly lower conductivity $\left(5.9 \times 10^{-5} \mathrm{~S} \mathrm{~m}^{-1}\right)$ than the CNT and the CB-filled wheat gluten at 0.4 vol\%. This was confirmed by several measurements at different relative humidities $(0-50 \% \mathrm{RH})$. The measured conductivity was in fact lower than that of the virgin WGG without any filler. For rGO/ polymer nanocomposites, $\phi_{\mathrm{p}}$ has been reported to be $0.2-2$ vol\%. ${ }^{\mathbf{4 3 4 4}}$ The currently lowest reported $\phi_{\mathrm{p}}$ values for a graphene-based composite are ca. 0.033 vol\% ${ }^{45}$ and 0.007 vol\%. ${ }^{46}$ The theoretical percolation threshold for soft oblate ellipsoids with an aspect ratio of 2000 is $\phi_{\mathrm{p}}=0.0637 \mathrm{vol} \%{ }^{47}$ A possible explanation for the more insulating nature of the rGO/WGG sample could be that there was a local accumulation of charges and/or an more tortuous path so that the sheets were unable to establish a network for percolation. Wang et al. ${ }^{48}$ reported that several rGO/poly(dimethyl siloxane) composites exhibited a lower conductivity than neat poly(dimethyl siloxane) at low field strength and suggested that it was related to a blockage of ion transport by the rGO network.

The conductivity of the foams with increasing compression is shown in Fig. 4b. The foams with the lowest concentration ( 0.15 vol\%) of CNTs were the most indifferent to the compression. However, samples with a higher content of CNTs showed a decreasing conductivity with increasing compression. This was most pronounced for CNT concentrations above the percolation threshold $(0.2 \mathrm{vol} \%)$ and is probably related to the deformation of the inherent organization of the CNTs that remained after the rapid freezing of the solution, i.e. the percolation network. In the case of the CB foams, the same dependence on the compression strain was observed at smaller carbon black particle contents, but at the highest contents the conductivity increased with increasing strain (Table 2, conductivity ratios $<1$ ), presumably due to a more "crowded" CB system, so that particles came closer together under compression, leading to improved particle-particle interaction and conductance. A similar increase in conductivity with compression of CB particles was earlier reported by Marinho et al. and Shang et al. ${ }^{\mathbf{4 9 , 5 0}}$

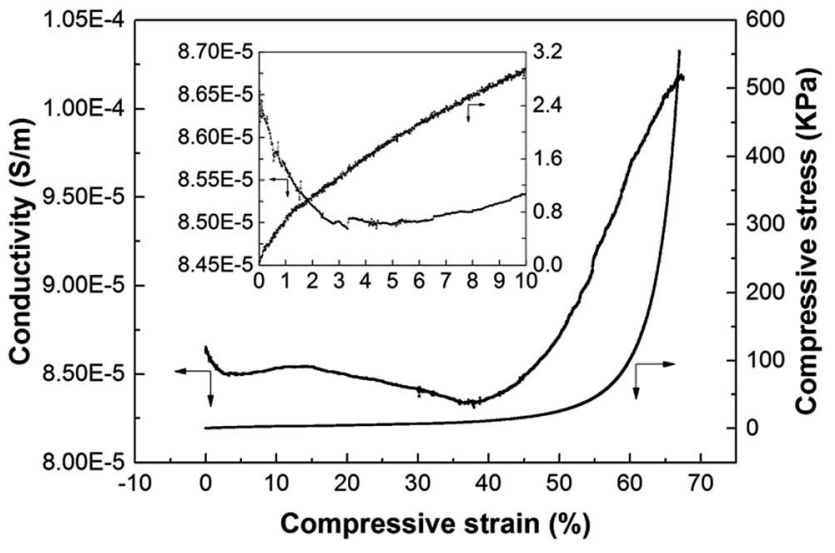

Fig. 5 The conductivity of the foam containing 0.39 vol\% rGO as a function of compressive strain. The inset shows the same curves within 0 to $10 \%$ strain.

Fig. 5 shows the conductivity of the rGO/WGG foam under compression. The conductivity first decreased with increasing compressive strain up to $\mathrm{ca}$. $40 \%$, and then increased (from 8.5 $\times 10^{-5}$ to $10 \times 10^{-5} \mathrm{~S} \mathrm{~m}^{-1}$ ) with increasing compressive strain from 40 to almost $70 \%$ strain. This behaviour was different from the CNT and CB systems and is presumably related to the morphology of the rGO sheets with their large sheet-like structure. After the collapse of the porous foam structure $(>40 \%$ strain) the rGO sheets more frequently came into contact and the conductivity of the samples increased (Fig. 5). The crosssection morphologies of compressed foams showed the evidence of structural collapse, i.e. broken cell walls, see Fig. S2 in ESI. $\uparrow$ However, due to its essentially poorly conducting nature before and after the compression, the origin of the conductivity change was not further explored since it may also relate to an accumulation difference and/or blockage/passage of ion transport by the rGO sheets, as suggested by Wang et al. ${ }^{48}$

\subsection{Microstructural wall characteristics}

Fig. 6a, b and c show cross-sections of the wall of WGG foams filled with ca. 0.4 vol\% of CNTs, CB and rGO, respectively. Voids were observed in the cell walls in all samples and in Fig. 6a the CNTs can be observed as nanofibers with a thickness of $c a$. $20 \mathrm{~nm}$. The CNTs occasionally bridged over the smaller voids (shown by white arrows) present in the cell walls. The majority of the CNTs did not however bridge across the larger cells observed in Fig. 3b-d and were mainly embedded in the cell walls. In the case of the CB foam, the carbon black particles were present as agglomerates embedded in the cell walls (Fig. 6b, within the white circles). It is suggested that this aggregated $\mathrm{CB}$ in the cell walls created regions with increased concentration of CB particles, which were interconnected with other local concentrations of the CB particles. This would explain why the entire sample was conductive although some areas of the wall surfaces did not show any CB particles. Fig. 6c inset shows the morphology of an individual pristine rGO sheet before mixing with WGG. The same buckled morphology was occasionally observed with isolated flat surface structures in the 

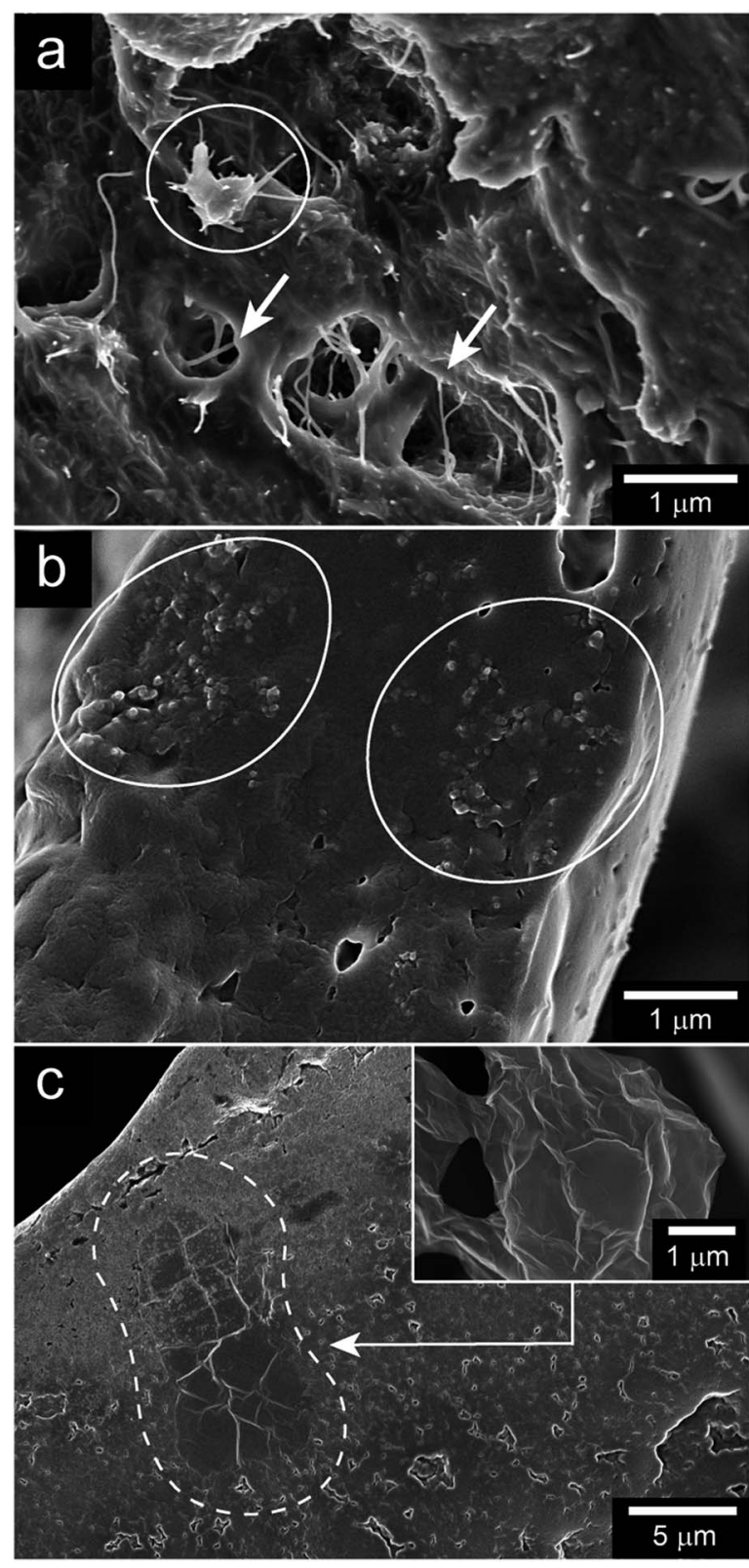

Fig. 6 SEM micrographs of plasticised-WG foam filled with (a) 0.41 vol\% CNT, (b) 0.44 vol\% CB, and (c) 0.39 vol\% rGO. The inset micrograph shows a rGO sheet without WGG.

walls of the WGG (see Fig. 6c). It was however very difficult to find more representative views of the rGO as dispersed sheets in the fractured walls of the WGG foam, since these sheets never bridged any of the open cells.

The inter-particle distances for $\mathrm{rGO}, \mathrm{CB}$ and CNTs were roughly estimated numerically by first placing $0.4 \mathrm{vol} \%$ particles on a mesh, then stochastically moving one of the particles in the grid to various non-colliding positions and finally calculating the average distance between the moved particle and its nearest neighbour. The CB particles were approximated as spheres (radius $10 \mathrm{~nm}$ ), the multi-wall CNTs as hollow cylinders (length $50 \mu \mathrm{m}$, radius $10 \mathrm{~nm}$, thickness $7 \mathrm{~nm}$ ) and the rGO as flakes (length $5 \mu \mathrm{m}$, width $3 \mu \mathrm{m}$, thickness $1.1 \mathrm{~nm}$ ). The theoretically estimated surface areas of the fillers were $1200 \mathrm{~mm}^{2}$ (CB), 900 $\mathrm{mm}^{2}$ (CNT) and $7300 \mathrm{~mm}^{2}$ (rGO) and the numbers of particles were $c a .9 .5 \times 10^{11}(\mathrm{CB}), 2.8 \times 10^{8}(\mathrm{CNT})$ and $2.4 \times 10^{8}(\mathrm{rGO})$. The number of CNTs and especially that of CB particles was significantly higher than the number of rGO sheets but the surface area of the rGO was nevertheless more dominant. In the foams, the average interparticle distance was approximately $18 \mathrm{~nm}(\mathrm{CB}), 0.2 \mathrm{~nm}(\mathrm{CNT})$ and $0.9 \mathrm{~nm}(\mathrm{rGO})$. The greater average interparticle distance in the rGO nanocomposite, as compared to the CNT nanocomposite, could possibly have contributed to its higher percolation threshold (above 0.4\%). Another explanation would be that strong sheet associations and possible stacking had occurred in the process of the wall formations. These associations have been assigned to the self-assemble of graphene sheets into nematic liquid crystals below the onset of percolation. ${ }^{51}$

\subsection{Mechanical properties}

Fig. 7 shows a representative stress-strain curve for the foam filled with CNT, and the inset shows the initially almost linear stress-strain behaviour at low strains $<10 \%$. The flat region of the main curve corresponds to cell collapse. The increase in stress at a strain greater than $60 \%$ shows the densification of the solid material after collapse of the foam. The compression modulus obtained from the initial linear slope (Fig. 7 inset) for all the materials is presented in Table 2. The modulus increased with increasing concentration of CNTs and the CNT foams showed overall a higher compression modulus than the foams filled with the same concentration of CB or rGO.

From a morphological point of view, the CNTs, with an aspect ratio of 2000, can be considered as reinforcing fibres..$^{52,53}$ The higher aspect ratio of the CNTs compared to that of the other fillers may therefore partially explain the increase in stiffness for the CNT/WGG samples. At the same time, it was however apparent that the modulus of the $\operatorname{CNT}(0.41)$ and CNT(0.49) foams were close to that of the virgin WGG foam $(\approx 33-36 \mathrm{kPa})$, while greater than those of $\operatorname{CNT}(0.1), \operatorname{CNT}(0.2)$, and $\mathrm{rGO}(0.4)$ and all of the CB foams $(\approx 13-26 \mathrm{kPa})$, see Table 2.

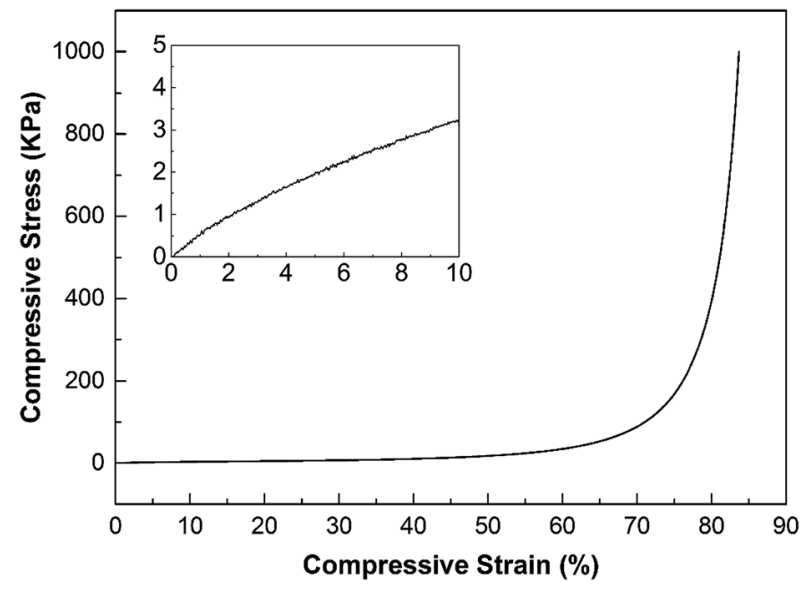

Fig. 7 The stress-strain curve of foam filled with 0.26 vol\% CNT, inset shows the same curve at the strain from $0 \%$ to $10 \%$. 
More detailed measurements on the approximate wall thicknesses for the different samples were therefore obtained. The cell wall thickness of the pure WGG was in average $25 \mu \mathrm{m}$. An increasing amount of CNTs resulted in an increase in wall thickness from ca. $12 \mu \mathrm{m}$ (CNT(0.26)) to ca. $25 \mu \mathrm{m}$ (CNT(0.49)) as the micrometre-sized cells decreased in size (see Section 3.1). This increase in wall thickness with increasing contents of CNTs was in contrast to the CB-filled WGG, which always showed thinner walls with greater amounts of $\mathrm{CB}$ added, resulting in ca. 5-6 $\mu \mathrm{m}$ wall thickness for the $0.44 \mathrm{vol} \% \mathrm{CB}$ (see Fig. 3e). The wall thickness for the rGO composite was $c a .10$ $\mu \mathrm{m}$. It is presently unclear to which extent the variations in cell wall thicknesses affected the load distribution over the compression tested samples, and/or if the thinner walls were more susceptible to irregularities in form of nano/submicrometric voids in the walls (Fig. 6). Such voids may originate from air trapped in the viscous solutions, before the freezing stage, which in turn may lower the stiffness of the cell walls. It is also clear that the mechanical properties (e.g. modulus) depended on the size, shape, and distribution of the cells, which varied significantly among the samples. Stress-strain curves for the 0.27 vol\% CB and 0.39 vol\% rGO are available, see ESI (Fig. S3†).

Cyclic compression tests were carried out to investigate the ability of the foams to recover after compression. Up to a compression of $\mathrm{ca} .10 \%$, the conductive foam was fully elastic with $100 \%$ recovery for the 0.41 vol\% CNTs. The foam also recovered after repeated $10 \%$ straining, the $10 \%$ strain cycle was repeated 5 times, and full recovery was observed for all cycles

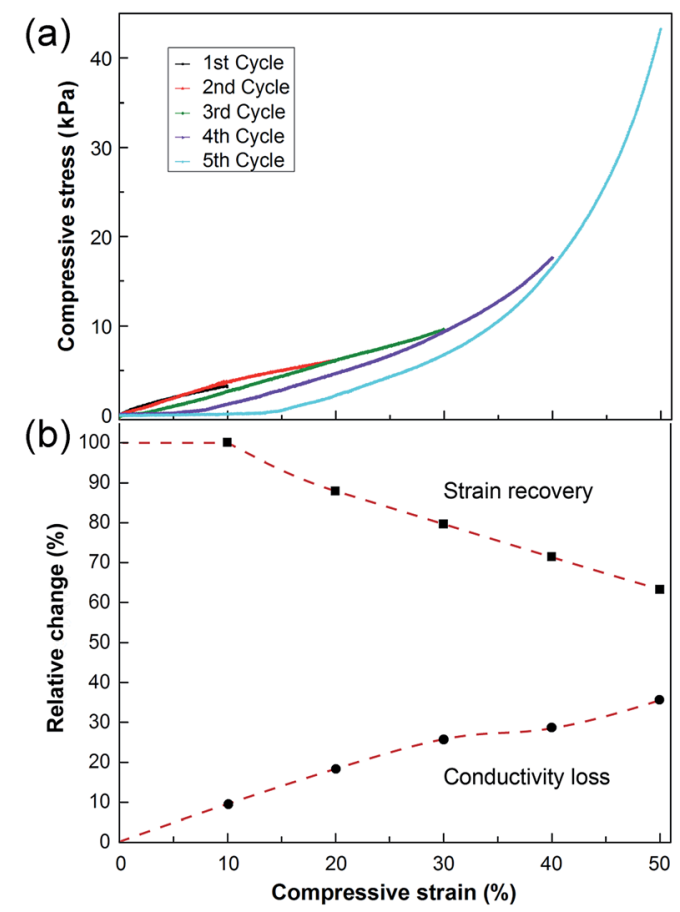

Fig. 8 (a) Compressive stress-strain curves of a cyclic compression test on a foam with 0.26 vol\% CNTs, (b) strain recovery of the foam after each compression cycle (filled squares), and conductivity loss of the foam after each compression cycle (filled circles).
(ESI, Fig. S4 $\dagger$ ). Fig. 8a shows the repetitive testing of a foam with 0.26 vol\% CNTs, i.e. a foam with a CNT amount immediately above the percolation threshold, which would be the most sensitive to changes in the percolating CNT network, (Fig. 4a). The test was conducted to investigate a possible reuse of a conductive biofoam exposed to consecutive and increasing compressions. The amount of recovery after each compression cycle decreased with increasing compressive strain $(>10 \%$ strain). Fig. 8b shows this decrease in recovery (filled squares) compared to the associated relative decrease in conductivity (filled circles) with each cycle. The decrease in conductivity above a peak strain of $10 \%$, was similar to that of the mechanical recovery, indicating a link between the destruction of the percolating network and the deformation of the cell walls. The decrease in conductivity below 10\% compressive strain indicated that the conductivity was not only related to the plastic deformation of the sample but also to the alteration of the percolating paths within the cell walls since the $10 \%$ purely elastic deformation resulted in a $c a .10 \%$ loss in conductivity. It is suggested that this is due to the high tensile strength of CNTs but very weak compressive strength of the individual tubes, causing them to bend even under elastic deformation.

\section{Conclusions}

Flexible and conductive biofoams based on wheat gluten have been prepared using a plasticiser and different carbon-based fillers (multiwall carbon nanotubes, carbon black and reduced graphene oxide). The cell structure was dependent on the type and content of the filler used, and more spherical cell shapes developed with increasing contents of conductive filler. The conductive WGG foams were fully elastic up to $10 \%$ compressive strain, but with increasing compression up to $50 \%$ strain, the recovery gradually decreased. A stiffening effect of CNTs was observed and the stiffness of the CNT/WGG foam increased with increasing CNT concentration. The foams filled with CNTs showed the highest conductivity followed by the CB and rGO at the same filler concentration of $0.4 \mathrm{vol} \%$. The high aspect ratio of CNTs led to a lower percolation threshold for the CNTs than for the CB and rGO systems. A decrease in conductivity with compression was apparent for the CNT/WGG biofoams, with the greatest decrease for the foams with higher fraction of CNT filler. Although a certain decrease in conductivity was observed post the fully elastic deformation regime (10\% strain), this decrease in conductivity was compensated for by the decrease in thickness, so that an essentially constant resistance was observed regardless of compression. This absence of a piezoresistive effect is a desired property in EMI and ESD applications where a constant resistance is important. ${ }^{54}$ The conductivity of the rGO-filled foam first decreased with the increasing of compressive strain up to $c a .40 \%$, and then increased with increasing strain from $40 \%$ to $c a$. $70 \%$. However, due to the essentially non-conductive nature of the rGO-filled biofoam in its non-deformed state (in comparison to the CNT or CB filled systems), and that a considerably higher fraction of rGO $(>0.4$ vol\%) would have been required for percolation, the rGO was not further explored. Finally, it could be concluded that all of 
the prepared WGG foams (containing CNTs and CB as a filler) conformed to the classifications (according to industrial standards: JESD625B, EIA-541 and ANSI/ESD-S541) $)^{4,5,55}$ of conductive materials for use in materials and tools for handling and packaging of sensitive electrical components (ESDS).

\section{Acknowledgements}

This work was financed by the Swedish Research Council Formas (No. 243-2011-1436). R. L. Andersson acknowledges the support from: European Institute of Innovation and Technology (EIT) - KIC InnoEnergy, Swedish Centre for Smart Grids and Energy Storage (SweGRIDS) and ABB AB.

\section{References}

1 Y. Yang, M. C. Gupta, K. L. Dudley and R. W. Lawrence, Adv. Mater., 2005, 17, 1999-2003.

2 Y. Yang, M. C. Gupta, K. L. Dudley and R. W. Lawrence, Nano Lett., 2005, 5, 2131-2134.

3 N. M. Julkapli, S. Bagheri and S. M. Sapuan, in Eco-friendly Polymer Nanocomposites: Processing and Properties, ed. K. V. Thakur and K. M. Thakur, Springer India, New Delhi, 2015, pp. 155-214.

4 JESD625B, Requirements for Handling Electrostatic DischargeSensitive (ESDS) Devices, 2012.

5 EIA-541, Packaging Material Standards for ESD Sensitive Items, 1988.

6 Z. Chen, C. Xu, C. Ma, W. Ren and H.-M. Cheng, Adv. Mater., 2013, 25, 1296-1300.

7 A. A. Al-Ghamdi, A. A. Al-Ghamdi, Y. Al-Turki, F. Yakuphanoglu and F. El-Tantawy, Composites, Part B, 2016, 88, 212-219.

8 M. Lobet, N. Reckinger, L. Henrard and P. Lambin, Nanotechnology, 2015, 26, 285702.

9 J. C. Wang, C. S. Xiang, Q. Liu, Y. B. Pan and J. K. Guo, Adv. Funct. Mater., 2008, 18, 2995-3002.

10 S. Sinha Ray, in Environmentally Friendly Polymer Nanocomposites, Woodhead Publishing, 2013, pp. 450-464.

11 M. M. Hamedi, A. Hajian, A. B. Fall, K. Håkansson, M. Salajkova, F. Lundell, L. Wågberg and L. A. Berglund, ACS Nano, 2014, 8, 2467-2476.

12 H. Ji, L. Zhang, M. T. Pettes, H. Li, S. Chen, L. Shi, R. Piner and R. S. Ruoff, Nano Lett., 2012, 12, 2446-2451.

13 Y. Qin, Q. Peng, Y. Ding, Z. Lin, C. Wang, Y. Li, F. Xu, J. Li, Y. Yuan, X. He and Y. Li, ACS Nano, 2015, 9, 8933-8941.

$14 \mathrm{H}$. Hu, Z. Zhao, R. Zhang, Y. Bin and J. Qiu, J. Mater. Chem. A, 2014, 2, 3756-3760.

15 M. T. Chen, L. Zhang, S. S. Duan, S. L. Jing, H. Jiang and C. Z. Li, Adv. Funct. Mater., 2014, 24, 7548-7556.

16 C. A. Martin, J. K. W. Sandler, M. S. P. Shaffer, M. K. Schwarz, W. Bauhofer, K. Schulte and A. H. Windle, Compos. Sci. Technol., 2004, 64, 2309-2316.

17 W. Bauhofer and J. Z. Kovacs, Compos. Sci. Technol., 2009, 69, 1486-1498.

18 H. Pang, L. Xu, D.-X. Yan and Z.-M. Li, Prog. Polym. Sci., 2014, 39, 1908-1933.
19 D.-X. Yan, H. Pang, B. Li, R. Vajtai, L. Xu, P.-G. Ren, J.-H. Wang and Z.-M. Li, Adv. Funct. Mater., 2015, 25, 559566.

20 A. V. Kyrylyuk, M. C. Hermant, T. Schilling, B. Klumperman, C. E. Koning and P. van der Schoot, Nat. Nanotechnol., 2011, 6, 364-369.

21 H. Pang, T. Chen, G. Zhang, B. Zeng and Z.-M. Li, Mater. Lett., 2010, 64, 2226-2229.

22 M. Yoonessi and J. R. Gaier, ACS Nano, 2010, 4, 72117220.

23 Y. Zhan, M. Lavorgna, G. Buonocore and H. Xia, J. Mater. Chem., 2012, 22, 10464-10468.

24 Z. M. Ali and L. J. Gibson, Soft Matter, 2013, 9, 1580-1588.

25 L. J. Sweetman, S. E. Moulton and G. G. Wallace, J. Mater. Chem., 2008, 18, 5417-5422.

26 Y. Zhou, S. Fu, Y. Pu, S. Pan and A. J. Ragauskas, Carbohydr. Polym., 2014, 112, 277-283.

27 T. Zimmermann, E. Pöhler and T. Geiger, Adv. Eng. Mater., 2004, 6, 754-761.

28 Z. Mohammadi, A. S.-M. Mesgar and F. Rasouli-Disfani, J. Mech. Behav. Biomed. Mater., 2016, 61, 590-599.

29 W. S. Veraverbeke and J. A. Delcour, Crit. Rev. Food Sci. Nutr., 2002, 42, 179-208.

30 Q. Wu, S. Yu, M. Kollert, M. Mtimet, S. V. Roth, U. W. Gedde, E. Johansson, R. T. Olsson and M. S. Hedenqvist, ACS Sustainable Chem. Eng., 2016, 4, 2395-2404.

31 T. O. J. Blomfeldt, R. Kuktaite, E. Johansson and M. S. Hedenqvist, Biomacromolecules, 2011, 12, 1707-1715.

32 P. U. Okoye and B. H. Hameed, Renewable Sustainable Energy Rev., 2016, 53, 558-574.

33 Q. Wu, R. L. Andersson, T. Holgate, E. Johansson, U. W. Gedde, R. T. Olsson and M. S. Hedenqvist, J. Mater. Chem. A, 2014, 2, 20996-21009.

34 T. O. J. Blomfeldt, R. T. Olsson, M. Menon, D. Plackett, E. Johansson and M. S. Hedenqvist, Macromol. Mater. Eng., 2010, 295, 796-801.

35 I. Rombouts, B. Lagrain, J. A. Delcour, H. Türe, M. S. Hedenqvist, E. Johansson and R. Kuktaite, Ind. Crops Prod., 2013, 51, 229-235.

36 C. T. F. Lo, K. Karan and B. R. Davis, Ind. Eng. Chem. Res., 2007, 46, 5478-5484.

37 D. S. McLachlan and G. Sauti, J. Nanomater., 2007, 2007, 9. 38 I. Balberg, Philos. Mag. B, 1987, 56, 991-1003.

39 S. K. H. Gulrez, M. E. Ali Mohsin, H. Shaikh, A. Anis, A. M. Pulose, M. K. Yadav, E. H. P. Qua and S. M. AlZahrani, Polym. Compos., 2014, 35, 900-914.

40 N. Shuhei, S. Kazuhiko, S. Goro and K. Keiichi, Jpn. J. Appl. Phys., 1997, 36, 5163.

41 N. Fritjof and U. Mikael, J. Phys. D: Appl. Phys., 2016, 49, 335303.

42 J. Krückel and D. W. Schubert, Eur. Polym. J., 2014, 53, 50-57. 43 J. R. Potts, D. R. Dreyer, C. W. Bielawski and R. S. Ruoff, Polymer, 2011, 52, 5-25.

44 T. Kuilla, S. Bhadra, D. Yao, N. H. Kim, S. Bose and J. H. Lee, Prog. Polym. Sci., 2010, 35, 1350-1375. 
45 H. Nassira, A. Sánchez-Ferrer, J. Adamcik, S. Handschin, H. Mahdavi, N. Taheri Qazvini and R. Mezzenga, Adv. Mater., 2016, 28, 6914-6920.

46 Z. Wang, X. Shen, N. M. Han, X. Liu, Y. Wu, W. Ye and J.-K. Kim, Chem. Mater., 2016, 28, 6731-6741.

47 E. J. Garboczi, K. A. Snyder, J. F. Douglas and M. F. Thorpe, Phys. Rev. E: Stat. Phys., Plasmas, Fluids, Relat. Interdiscip. Top., 1995, 52, 819-828.

48 Z. Wang, J. K. Nelson, H. Hillborg, S. Zhao and L. S. Schadler, Adv. Mater., 2012, 24, 3134-3137.

49 B. Marinho, M. Ghislandi, E. Tkalya, C. E. Koning and G. de With, Powder Technol., 2012, 221, 351-358.
50 S. Shang, Y. Yue and X. Wang, Rev. Sci. Instrum., 2016, 87, 123910.

51 J. K. Yuan, et al., Nat. Commun., 2015, 6, 8700.

52 S. R. Shin, S. J. Park, S. G. Yoon, C. K. Lee, K. M. Shin, B. K. Gu, M. K. Shin, M. S. Kim, Y. J. Kim and S. J. Kim, Mater. Res. Soc. Symp. Proc., 2011, 915, 11-16.

53 S. R. Popuri, R. Frederick, C.-Y. Chang, S.-S. Fang, C.-C. Wang and L.-C. Lee, Desalin. Water Treat., 2014, 52, 691-701.

54 N. F. Colaneri and L. W. Schacklette, IEEE Trans. Instrum. Meas., 1992, 41, 291-297.

55 ANSI/ESD-S541, Packaging Materials for ESD Sensitive Items, 2008. 